\title{
The Effect of Intra- vs. Extramedullary Tibial Guides on the Alignment of Lower Extremity and Functional Outcomes following Total Knee Arthroplasty: A Randomized Clinical Trial
}

\section{Mohammadreza Razzaghof}

Joint Reconstruction Research Center (JRRC), Tehran University of Medical Sciences

SM Javad Mortazavi ( $\sim$ smjmort@yahoo.com )

Joint Reconstruction Research Center (JRRC), Tehran University of Medical Sciences

Alireza Moharrami

Joint Reconstruction Research Center (JRRC), Tehran University of Medical Sciences

Pouya Tabatabaei Irani

Joint Reconstruction Research Center (JRRC), Tehran University of Medical Sciences

Abbas Noori

Joint Reconstruction Research Center (JRRC), Tehran University of Medical Sciences

\section{Research Article}

Keywords: Total knee arthroplasty, Intramedullary guide, Extramedullary guide, Functional outcome

Posted Date: May 27th, 2021

DOI: https://doi.org/10.21203/rs.3.rs-505262/v1

License: (c) (i) This work is licensed under a Creative Commons Attribution 4.0 International License.

Read Full License 


\section{Abstract}

Background: Total knee arthroplasty (TKA) has been known as a definitive treatment of advanced knee osteoarthritis. Both intra- (IM) and extramedullary (EM) tibial guides have been used to restore the desired extremity alignment. However, controversy exists regarding the superiority of either technique. We aimed to compare their functional outcomes and accuracy in providing neutral alignment after TKA.

Methods: In a randomized, double-blinded clinical trial, we studied 98 patients undergoing primary TKA in two groups of IM and EM. We measured the medial proximal tibial angle (MPTA), varus angle (VA), and joint-line convergence angle with normal ranges of $90^{\circ} \pm 3^{\circ}, 0-2^{\circ}$, and $0 \pm 3^{\circ}$, respectively, on a three-joint alignment view after three months. We also assessed functional outcomes at the last follow-up. Finally, we compared these outcomes between groups.

Results: Eighty-four patients (IM=42, EM=42) were included in the final analysis (16 males, 68 females; mean age: $63.9 \pm 8.6$ years; mean follow-up: $27 \pm 2.9$ months). The mean postoperative alignment angles showed no significant difference, although MPTA outliers were significantly more frequent in the EM group ( $26.2 \%$ vs. $9.5 \%$ in $I M, P=0.04)$. None of the functional outcomes showed a significant difference between groups. However, the mean ROM increase was significantly higher in VAs within $\pm 3^{\circ}$ of normal than those outside it (30.8 vs. 27.4 , respectively; $P=0.039$ ).

Conclusions: We conclude that both techniques were not different in terms of the mean alignment angles and functional outcomes. However, fewer MPTA outliers can be seen with IM. A postoperative mechanical axis within $\pm 3^{\circ}$ of neutral can result in a more ROM increase after one year.

Trial registration: IRCT, IRCT20160809029286N5. Registered 21 April 2020, https://www.irct.ir/trial/46976

\section{Background}

Total knee arthroplasty (TKA) has been known as an end to analgesics use in patients with knee osteoarthritis and as a means of restoring the physical activity and function (1). Several factors are influential in the long term outcome of a TKA, including patient selection, the prosthesis of use, and surgical technique (2). The surgical technique is of great importance among knee surgeons as it should implement proper lower extremity alignment for a good long-term prognosis (1-3). Several studies have reported poor outcomes of the lower extremity malalignment, as prosthesis mispositioning can lead to loosening, recurrence of pain, and compromised physical performance (4-8).

Among surgical techniques, both intra- (IM) and extramedullary (EM) tibial guides have been used to provide the desired lower extremity alignment. However, there are still controversies over the outcome of TKA using either technique to provide a neutral alignment $(1,9)$. Some studies have preferred one for providing a more accurate tibial alignment (9-11), while the others found no significant difference between either technique (1,12-14). Many studies have demonstrated that most knees are suitable for hath torhninuoc hnwavar thic ic nnt alwave the case. EM guides are unreliable in cases of soft tissue or Loading [MathJax]/jax/output/CommonHTML/jax.js 
ankle abnormality, whereas IM technique is not preferred in patients with excessive tibial bowing, previous fracture, or retained metalwork (9). Moreover, the functional outcomes following both techniques were compared only by few studies $(10,15)$. In the present study, we decided to compare the accuracy of $\mathrm{IM}$ and EM techniques in providing neutral lower extremity alignment in patients undergoing TKA. We also compared the functional outcomes following TKA between both techniques.

\section{Methods}

This randomized clinical trial was designed and reported based on the Consolidated Standards of Reporting Trials (CONSORT) principles. The study protocol was reviewed and approved by the institutional review board of our university. The Ethics Committee Board declared no ethical concern in the current study. All the patients were informed of the fact that the results of this study are to be published in scientific medical journals and gave their informed consent. Moreover, the Ethics Committee Board allowed the publishing of study results. This trial was registered in 21/04/2020 in the clinical trial registry of our country with the identification number of IRCT20160809029286N5.

\subsection{Study design and participants}

This study was a randomized controlled clinical trial. A total of 98 consecutive patients who met the eligibility criteria of the study were enrolled from the orthopedic clinic of our institute. The inclusion criteria were patients with primary knee osteoarthritis indicated for TKA, who had a varus or neutral knee alignment. The exclusion criteria were: 1 . hemophilia, 2 . inflammatory knee arthritis like rheumatoid arthritis, 3. previous tibial fracture, 4 . genu valgum, and 5. inadequate radiographs.

\subsection{Study protocol and interventions}

The enrolled patients were admitted and underwent pre-operative imaging and TKA according to the study protocol. The patients participated voluntarily and had signed the informed consent formula. An assistant researcher did the data collection and clinical assessment of the functional outcomes following the surgery. A single experienced knee surgeon performed all the TKAs.

\subsubsection{Imaging protocol}

A full standing three-joint alignment view (3-JV) of the lower limb together with the anteroposterior and lateral knee projections were performed for each patient before and three months after the surgery. For the 3-JV, the patient must stand with the knees in full extension and no aid. The patella should face forward in the direction of the x-ray beam. The collimator was set superiorly on the iliac crest and inferiorly on the sole. The radiograph is composed automatically after three projections done by CARESTREAM DRX-Evolution device (Carestream Health Inc., US). The alignment angles of the lower limb were measured on 3-JV using the MediCAD software by a single experienced knee surgeon. The acceptable (inlier) range for MPTA was considered $90^{\circ} \pm 3^{\circ}, \mathrm{JLCA} 0^{\circ}$ to $2^{\circ}$, and VA $0 \pm 3^{\circ}$.

\subsubsection{Suraical techniaue


After spinal anesthesia and standard prepping and draping, TKA was performed using a standard anterior midline incision with the anteromedial approach. A pneumatic tourniquet had been applied above the knee prior to the operation. Releasing the medial soft tissue of the knee, we subluxated the patella and subsequently exposed the proximal tibia. For femoral cuts, we used an intramedullary jig. To perform tibial cuts, we used an intra- or extramedullary jig in each group accordingly. The entry point for IM jig was the center of the tibial axis, according to preoperative planning, on tibial plateau anterior to the insertion of the anterior cruciate ligament. The EM jig was mounted on the leg parallel with the tibial axis and leveled proximally with the tibial crest in the coronal plane and distally with the talus center in the axial plane ( $3^{\mathrm{mm}}$ medial to ankle center). The tibial bone cut was done with a posterior slope of $3^{\circ}$. The tibial component was placed in a $3^{\circ}$ external rotation. In all the patients, a cemented posterior stabilized

(PS) NexGen $\odot$ LPS-Flex Knee prosthesis (Zimmer Biomet ${ }^{\circledR}$, Warsaw, Indiana, US) was used. Finally, standard wound closure and care were done.

\subsection{Outcomes}

The primary outcome measures were the radiologic and functional outcomes, which were assessed both pre- and postoperatively. The radiologic outcomes included the medial proximal tibial angle (MPTA), mechanical femoral mechanical tibial or varus angle (MFMTA or VA), and joint-line convergence angle (JLCA). The functional outcomes included the knee society score (KSS), functional knee society score (fKSS), pain visual analog scale (VAS), and the measurement of knee range of motion (ROM). The postoperative assessment of the radiologic and functional outcomes was at three months, and the last follow-up visit, respectively.

\subsection{Sample size}

The sample size was calculated based on the study of Chin et al. (16) that compared the three techniques of IM, EM tibial guides, and computer-navigated surgery in the patients who underwent TKA. We used the risk ratio of postoperative MPTA angle outliers ( $p_{1}=43.34 \% \mathrm{IM}$ vs. $p_{2}=13.34 \% \mathrm{EM}$ ) as a reference value for power analysis and assumed a $\beta$ value of $20 \%$ and a of $5 \%$. We found that 35 patients per each group (70 patients in total) were required to achieve statistical significance. The calculations are presented below. We considered a sample size of at least 90 to compensate for possible losses during follow-up.

$$
\begin{aligned}
& n_{1}=\frac{\left\{z_{1-\frac{\alpha}{2}} * \sqrt{\bar{p} * \bar{q} *\left(1+\frac{1}{k}\right)}+z_{1-\beta} * \sqrt{p_{1} * q_{1}+\left(\frac{p_{2}+q_{2}}{k}\right)}\right\}^{2}}{\Delta^{2}} \\
& q_{1}=1-p_{1}, q_{2}=1-p_{2}, \bar{p}=\frac{p_{1}+k p_{2}}{1+k}, \bar{q}=1-\bar{p}, \Delta=\left|p_{1}-p_{2}\right|, k=\frac{n_{2}}{n_{1}}=1
\end{aligned}
$$

\subsection{Randomization and blinding}


The patients were randomly assigned to two groups of intramedullary $(n=48)$ and extramedullary $(n=$ 48) based on the tibial guide used during the surgery. The patients had an equal chance of being randomly assigned to each of the two arms of the study. The randomization of the patients was done using the permuted balanced block method. Six blocks of four were assumed, and the patients were divided into 21 sequentially numbered groups. The groups were randomized using a list of random numbers generated by Microsoft Excel 365, and the patients of each group were allocated to each intervention arm accordingly. The randomization sequence was concealed before the enrollment until the patient was transferred to the operation room. An independent researcher, who was not involved in the process of data collection and outcome assessment, performed the randomization. This study was tripleblinded, as neither the patient nor the assistant researcher nor the analyzer researcher was aware of the technique used during the surgery.

\subsection{Data analysis}

Data analysis was done by SPSS 25.0 software (SPSS Inc., Chicago, Illinois, US). The normality of the variables was tested by the skewness-kurtosis and Spearman Tests. The Student's independent $t$-test was used to compare continuous outcome variables such as the alignment angles in both groups. The chisquare and Fischer's Exact tests were used to compare the nominal outcome variables. The significance level was considered 0.05 .

\section{Results}

A total of 98 patients who underwent TKA using either EM or IM tibial guide were enrolled in the study from September 2018 to May 2019. Two patients were excluded as they declined to participate in the study. The patients were randomized into two groups of IM $(n=48)$ and EM $(n=48)$ tibial guides. All the patients received the allocated intervention. There was no loss to follow-up. Twelve patients were excluded from the final analysis due to inadequate postoperative radiographs. The CONSORT flow chart is shown in Fig. 1.

The mean age and body mass index (BMI) were $63.9 \pm 8.6$ and $29.6 \pm 4.8$, respectively. There were 16 (19\%) males and $68(81 \%)$ females. The mean follow-up duration was $27 \pm 2.9$ months (range $=7-19)$. As seen in Table 1, both groups had the same and matched demographics $(P>0.05)$. 
Table 1

Demographic data of the patients

\begin{tabular}{|c|c|c|c|c|c|}
\hline & & $\begin{array}{l}\mathrm{IM} \\
(\mathrm{N}=42)\end{array}$ & $\begin{array}{l}E M \\
(N=42)\end{array}$ & $p$ Value & $\begin{array}{l}\text { Total } \\
(\mathrm{N}=84)\end{array}$ \\
\hline \multicolumn{2}{|c|}{ Age (years) } & $66.0 \pm 9.8$ & $62.4 \pm 7.4$ & $0.56^{\star}$ & $63.9 \pm 8.6$ \\
\hline \multirow[t]{2}{*}{ Sex } & Male & 10 & 6 & \multirow[t]{2}{*}{0.71 ** } & 16 \\
\hline & Female & 32 & 36 & & 68 \\
\hline \multicolumn{2}{|c|}{$\operatorname{BMI}\left(\mathrm{Kg} / \mathrm{m}^{2}\right)$} & $29.5 \pm 5.1$ & $29.6 \pm 4.7$ & $0.92^{*}$ & $29.6 \pm 4.8$ \\
\hline \multicolumn{2}{|c|}{ Follow-up (months) } & $26.8 \pm 1.9$ & $27.2 \pm 3.7$ & $0.58 *$ & $27 \pm 2.9$ \\
\hline
\end{tabular}

As seen in Table 2, the mean of the lower limb alignment angles, including MPTA, JLCA, and VA, was compared between both groups preoperatively and at three months postoperatively. Our data showed that there was no significant difference between EM and IM groups in terms of the lower limb alignment angles $(P>0.05)$.

Table 2

The preoperative vs. postoperative alignment angles of lower extremity (MPTA, JLCA, and VA) in both groups of the study

\begin{tabular}{|c|c|c|c|c|c|}
\hline & & $\begin{array}{l}\text { Intramedullary } \\
\text { Mean (SD) }\end{array}$ & $\begin{array}{l}\text { Extramedullary } \\
\text { Mean (SD) }\end{array}$ & $p$ Value* & $\begin{array}{l}\text { Total } \\
\text { Mean (SD) }\end{array}$ \\
\hline \multirow[t]{2}{*}{ MPTA } & Preop & $84.3( \pm 2.9)$ & $84.8( \pm 4.1)$ & 0.48 & $84.6( \pm 3.7)$ \\
\hline & Postop & $88.9( \pm 2.1)$ & $88.4( \pm 2.9)$ & 0.42 & $88.6( \pm 2.6)$ \\
\hline \multirow[t]{2}{*}{ JLCA } & Preop & $9.0( \pm 5.0)$ & $9.2( \pm 8.2)$ & 0.88 & $9.1( \pm 7)$ \\
\hline & Postop & $0.5( \pm 1.2)$ & $0.2( \pm 0.5)$ & 0.12 & $0.3( \pm 0.9)$ \\
\hline \multirow[t]{2}{*}{ VA } & Preop & $15.7( \pm 7.9)$ & $14.3( \pm 7.5)$ & 0.38 & $14.9( \pm 7.7)$ \\
\hline & Postop & $3.5( \pm 2.7)$ & $3.4( \pm 2.2)$ & 0.86 & $3.4( \pm 2.4)$ \\
\hline
\end{tabular}

As mentioned earlier, we considered the normal range (inliers) for MPTA, JLCA, and VA as $90^{\circ} \pm 3^{\circ}, 0$ to $2^{\circ}$, and $0 \pm 3^{\circ}$, respectively. The outlier cases of MPTA, JLCA, and VA are presented in Table 3 . The postoperative MPTA outliers were significantly more frequent in the EM than the IM group $(26.2 \%$ vs. $9.5 \%, P=0.04)$. However, no significant difference was found between the JLCA and VA outliers of the 
Table 3

The MPTA, JLCA, and VA outliers following TKA in both groups of the study

\begin{tabular}{|llll|}
\hline & $\begin{array}{l}\text { Intramedullary } \\
\text { Frequency (ratio \%) }\end{array}$ & $\begin{array}{l}\text { Extramedullary } \\
\text { Frequency (ratio \%) }\end{array}$ & p Value* \\
\hline MPTA $^{1}$ Postop & $4(9.5 \%)$ & $11(26.2 \%)$ & 0.04 \\
\hline JLCA $^{2}$ Postop & $3(7.1 \%)$ & $1(2.4 \%)$ & 0.306 \\
\hline VA $^{3}$ Postop & $16(38.1 \%)$ & $19(45.2 \%)$ & 0.507 \\
\hline${ }^{1} 90^{\circ} \pm 3^{\circ}$ range was considered as normal. & \\
${ }^{2} 0-2^{\circ}$ range was considered as normal. & \\
${ }^{3} 0 \pm 3^{\circ}$ range was considered as normal. & \\
$*$ &
\end{tabular}

As shown in Table 4, our data showed that body weight was significantly lower in the outliers of the EM group than that of the inliers $(71.5 \pm 10.2$ vs. $80.2 \pm 10.6, P=0.02)$. The same result was found in all the patients $(71.7 \pm 8.5$ vs. $79.1 \pm 12.1, P=0.04)$. However, no significant difference was found between the outliers and inliers of the IM group $(72.5 \pm 2.0$ vs. $78.2 \pm 14.5, P=0.43)$. The risk ratio of MPTA outliers was $5 / 32$ and $5 / 10$ in patients of EM group with weights $>70$ and $\leq 70 \mathrm{Kg}$, respectively, the difference of which was significant (chi-square test, $P=0.03$ ). However, it was $3 / 27$ (weight $>70 \mathrm{Kg}$ ) and 1/15 (weight $\leq 70 \mathrm{Kg}$ ) in patients of IM group showing no significant difference (Fischer's Exact test, $P=1.0$ ). The mean BMI was also compared but showed no significant difference between the MPTA outliers and inliers of IM (29.7 vs. 28.3 , respectively, $P=0.62)$ and $E M\left(30.1\right.$ vs. $28.6 \mathrm{Kg} / \mathrm{m}^{2}$, respectively, $\left.P=0.37\right)$ groups. Moreover, the risk ratio of MPTA outliers was $1 / 8$ and 9/34 in patients of EM group with $\mathrm{BMI} \geq 35$ and $<35 \mathrm{Kg} / \mathrm{m}^{2}$, respectively, with no significant difference (Fischer's Exact test, $P=0.66$ ). It was $0 / 8$ $\left(\mathrm{BMI} \geq 35 \mathrm{Kg} / \mathrm{m}^{2}\right)$ and $4 / 34\left(\mathrm{BMI}<35 \mathrm{Kg} / \mathrm{m}^{2}\right)$ in patients of IM group, respectively, with no significant difference as well (Fischer's Exact test, $P=0.58$ ). 
Table 4

The mean patients' weight $(\mathrm{Kg})$ of the outliers vs. inliers based on the postoperative MPTA angle following TKA

\begin{tabular}{|llll|}
\hline & $\begin{array}{l}\text { Outliers } \\
\text { Mean (SD) }\end{array}$ & $\begin{array}{l}\text { Inliers } \\
\text { Mean (SD) }\end{array}$ & $\begin{array}{l}\text { Pvalue* } \\
\text { Mean (SD) }\end{array}$ \\
\hline Intramedullary & $72.5( \pm 2.0)$ & $78.2( \pm 14.5)$ & 0.43 \\
\hline Extramedullary & $71.5( \pm 10.2)$ & $80.2( \pm 10.6)$ & 0.02 \\
\hline Total & $71.7( \pm 8.5)$ & $79.1( \pm 12.1)$ & 0.04 \\
\hline $\begin{array}{l}190^{\circ} \pm 3^{\circ} \text { range was considered as normal. } \\
\text { * independent } t \text { test }\end{array}$ & \\
\hline
\end{tabular}

The patients were followed for an average of $13 \pm 2.9$ months. The functional outcome measures, including KSS, fKSS, VAS, and the knee ROM were measured for all the patients preoperatively and in the last follow-up visit, as seen in Table 5. However, no significant difference was found between the EM and IM groups in terms of these outcome measures at a mean follow-up of $27 \pm 2.9$ months $(P>0.05)$. The preoperative and postoperative VAS pain scores showed no significant difference as well $(1.09 \pm 1 \mathrm{vs.} 1.2$ $\pm 1, P=0.45)$.

Table 5

The preoperative vs. postoperative of KSS, FKSS, VAS score, and the increase in ROM in both groups of the study

\begin{tabular}{|llllll|}
\hline & & $\begin{array}{l}\text { Intramedullary } \\
\text { Mean (SD) }\end{array}$ & $\begin{array}{l}\text { Extramedullary } \\
\text { Mean }(\text { SD })\end{array}$ & $p$ Value* & $\begin{array}{l}\text { Total } \\
\text { Mean (SD) }\end{array}$ \\
\hline \multirow{2}{*}{ KSS } & Preop & $34.2( \pm 12.3)$ & $32.0( \pm 8.7)$ & 0.3 & $32.9( \pm 10.4)$ \\
\cline { 2 - 6 } & postop & $91.1( \pm 5)$ & $90.4( \pm 4.8)$ & 0.45 & $90.7( \pm 4.9)$ \\
\hline \multirow{2}{*}{ VAS Pain } & Preop & $30.1( \pm 7.2)$ & $29.7( \pm 3.9)$ & 0.76 & $29.9( \pm 5.5)$ \\
\cline { 2 - 6 } & postop & $91.1( \pm 4.2)$ & $90.7( \pm 4.5)$ & 0.67 & $90.9( \pm 4.4)$ \\
\hline \multirow{2}{*}{ Increase in ROM (postop) } & $30.1( \pm 9.3)$ & $27.6( \pm 5)$ & 0.95 & $28.7( \pm 7.2)$ \\
\hline * independent $t$ test & & & & & \\
\hline
\end{tabular}

The functional outcomes were also compared between the inlier and outlier ranges of postoperative VA, which demonstrated no significant difference except for the increase in ROM. In those patients with a VA within $\pm 3^{\circ}$ of normal, the mean increase in ROM following TKA was significantly higher than that of Loading [MathJax]/jax/output/CommonHTML/jax.js vs. 27.4, respectively; $\mathrm{P}=0.039$ ) (Fig. 2). 


\section{Discussion}

The controversy over IM and EM tibial guides has existed in the literature since the very introduction of both techniques $(1,9,17)$. Based on a systematic review, the published literature includes nearly twenty original articles on this issue over the last three decades. Of these, $52.6 \%$ believed the two techniques had comparable accuracy, $36.8 \%$ preferred IM guides, and 10.5\% found EM guides more accurate (1). However, not all these studies had sufficient sample size, proper methodology, or reported all the important radiologic or functional outcomes. Accordingly, only six of them were eligible for the metaanalysis by Zeng et al. (1). The results of our trial showed no significant difference between the accuracy of the two techniques in terms of the mean post-op MPTA, JLCA, and VA values. However, the number of MPTA outliers was significantly lower in the IM group. The number of outliers for JLCA and VA showed no significant difference. Both groups demonstrated good functional outcomes with no significant difference.

Three radiologic measures have been so far discussed in the literature to compare the accuracy of tibial alignment following IM and EM techniques. These include the MPTA, VA, and tibial slope angles. The mean values of these measures were not significantly different between both techniques in many of the studies. However, it should be noted that the mean value overlooks the presence of outliers, which might be significant when comparing both techniques, noted only by a few studies.

The MPTA is the angle between the tibial mechanical axis and the articular surface of the tibial component in AP knee projection. Different studies have used various terms for it, such as the frontal tibial component angle (1), coronal tibiofemoral angle (15), and tibial component angle (9). Although the mean MPTA reported by Chin and Reed et al. was not significant between both techniques, the two studies were contradictory in terms of the relative risk of outliers. The EM-to-IM risk ratio of outliers was 0.31 versus 2.35 in the studies of Chin and Reed et al., respectively $(9,16)$. However, the meta-analysis of the pooled data of both studies (20/76 EM vs. $21 / 84 \mathrm{IM}$ ) showed no significant difference (1). Our study also showed also no significant difference between the mean post-op MPTAs of both techniques, although the difference between the outliers was significant (11/42 EM vs. 4/42 IM, EM-to-IM risk ratio = 2.75), consistent with the results of Reed et al. study.

The VA or MFMTA is the angle between the mechanical axis of femur and tibia in AP radiograph, which has also been designated as the mechanical axis angle $(1,16,18)$. Neither the mean value nor the outlier relative risk of VA was significantly different between the two techniques in the studies of Chin, Blakeney, and Kroon et al. $(16,18,19)$. The meta-analysis of pooled data (31/83 EM vs. 30/89 IM) demonstrated the same result (1). Our result (19/42 EM vs. 16/42 IM) is also consistent with the results of these studies. The tibial slope is another angle, which also showed no significant difference in the metaanalysis study of Zeng et al. (1). We did not report this measure. However, we compared post-op JLCA between the two techniques, not reported before, which also showed no significant difference.

In the ancillary analysis of outlier data, we found that the mean weight of the patients was significantly Loading [MathJax]/jax/output/CommonHTML/jax.js liers of the EM group, although no such difference was seen 
in the IM group. However, BMI showed no significant difference between the outliers and inliers of each group. We found no relevant data in the previous studies comparing both techniques. Nevertheless, a recent study by Compton et al. showed that BMI did not influence the postoperative MPTA following TKA using EM guides. They found no difference between the MPTA outlier (defined as outside $\pm 5^{\circ}$ ) ratio of patients with a BMI $<35(2 / 100)$ and $\geq 35(2 / 62)(20)$. It was also not significantly different between those with a $\mathrm{BMI}<35(9 / 34)$ and $\geq 35(1 / 8)$ in EM group of our study, which is consistent with the findings of Compton et al. However, the significantly lower weights of the patients, who underwent TKA using an EM guide and have a final MPTA outside the normal range, might indicate that thinner legs are associated with a higher chance of error in the appropriate mounting of an EM jig and doing tibial cuts. It might be due to the underestimation of a thinner leg by the surgeon, as he usually expects the obese legs to be a challenge. We think further comparative studies between both techniques are needed, which focus particularly on the length and girth of the leg to determine the effect of these parameters on the final lower extremity alignment.

The functional outcomes following TKA using either IM or EM techniques have been compared only by a few studies. Cashman et al. reported the SF-36 and WOMAC scores in 103 Triathlon TKAs (36 IM vs. 67 EM), which were not significantly different between both techniques at six months (10). Blakeney et al., in their second study in 2014, compared the physical and mental component scores of SF-12 and the Oxford Knee Score (OKS) between 107 patients undergoing TKA using IM, EM, and computer-assisted techniques at a median follow-up of 46 months. None of these measures differed significantly between IM and EM techniques, although OKS adjusted for sex and age showed a difference, close to the significance level, between computer-assisted and conventional techniques (15). In our study, the postoperative values of KSS, fKSS, pain VAS, and the increase in ROM were not significantly different between IM and EM groups at a mean follow-up of $27 \pm 2.9$ months. However, a postoperative mechanical axis within $\pm 3^{\circ}$ of neutral was associated with a more increase in knee ROM than that outside this range (Fig. 2).

\section{Conclusions}

In this randomized clinical trial, no significant difference was found between IM and EM techniques for TKA in terms of the mean postoperative lower extremity alignment angles, including MPTA, JLCA, and VA. However, the IM technique was associated with fewer postoperative MPTA outliers. Both techniques were found equal regarding the good functional outcomes seen with both. In general, a postoperative mechanical axis within $\pm 3^{\circ}$ of neutral was associated with a more increase in ROM at mean 27-month follow-up.

\section{Abbreviations}

TKA: Total knee arthroplasty 
EM: extramedullary (guide)

CONSORT: Consolidated Standards of Reporting Trials

3-JV: three-joint alignment view

PS: posterior stabilized (prosthesis)

MPTA: medial proximal tibial angle

MFMTA: mechanical femoral mechanical tibial

VA: varus angle

JLCA: joint-line convergence angle

KSS: knee society score

fKSS: functional knee society score

VAS: visual analog scale (of pain)

ROM: range of motion

BMI: body mass index

\section{Declarations}

Ethics approval and consent to participate: All the patients participated voluntarily in this study and had signed the informed consent formula. The Ethics Committee Board of Tehran university of medical sciences approved of the methodology and declared no ethical concern in this study. All the methods were performed in accordance with the guidelines and regulations of the institutional review board of our university.

Consent for publication: All the patients were informed of the fact that the results of this study are to be published in scientific medical journals and gave their informed consent. Moreover, the Ethics Committee Board allowed the publishing of study results.

Availability of data and materials: The datasets of the current study are available from the corresponding author on reasonable request.

Competing interests: The authors declare that they have no conflict of interest.

Funding: This research did not receive any specific grant from funding agencies in the public, commercial, or not-for-profit sectors. 
Authors' contributions: All the authors have contributed to this research. Professor SMJ.M. was the senior surgeon, who designed and supervised the study process, and performed the surgeries. M.R. performed the randomization of the patients, data collection, and wrote the final manuscript of the study. A.M. did the data analysis and measured the radiographic parameters of the study. P. TI. helped in reporting the results and data collection. A.N. was our fellowship knee surgeon, who helped both in performing the surgeries and follow-up of the patients in follow-up clinic. All the authors reviewed and approved of the final manuscript.

Acknowledgements: None declared.

\section{References}

1. Zeng HB, Ying XZ, Chen GJ, Yang XQ, Lin DD, Li ZJ, et al. Extramedullary versus intramedullary tibial alignment technique in total knee arthroplasty: A meta-analysis of randomized controlled trials. Clinics. 2015;70(10):714-9.

2. Maestro A, Harwin SF, Sandoval MG, Vaquero DH, Murcia A. Influence of intramedullary versus extramedullary alignment guides on final total knee arthroplasty component position: a radiographic analysis. The Journal of arthroplasty. 1998;13(5):552-8.

3. Ritter MA, Faris PM, Keating EM, Meding JB. Postoperative alignment of total knee replacement. Its effect on survival. Clinical orthopaedics and related research. 1994(299):153-6.

4. Sharkey PF, Hozack WJ, Rothman RH, Shastri S, Jacoby SM. Why are total knee arthroplasties failing today? Clinical Orthopaedics and Related Research®. 2002;404:7-13.

5. Jeffery RS, Morris RW, Denham RA. Coronal alignment after total knee replacement. The Journal of bone and joint surgery British volume. 1991;73(5):709-14.

6. Incavo SJ, Wild JJ, Coughlin KM, Beynnon BD. Early revision for component malrotation in total knee arthroplasty. Clinical Orthopaedics and Related Research®. 2007;458:131-6.

7. Fang DM, Ritter MA, Davis KE. Coronal alignment in total knee arthroplasty: just how important is it? The Journal of arthroplasty. 2009;24(6):39-43.

8. Berger R, Crossett L, Jacobs J, Rubash H. Malrotation causing patellofemoral complications after total knee arthroplasty. Clinical Orthopaedics and Related Research ${ }^{\circledR} .1998 ; 356: 144-53$.

9. Reed M, Bliss W, Sher J, Emmerson K, Jones S, Partington P. Extramedullary or intramedullary tibial alignment guides: a randomised, prospective trial of radiological alignment. The Journal of bone and joint surgery British volume. 2002;84(6):858-60.

10. Cashman JP, Carty FL, Synnott K, Kenny PJ. Intramedullary versus extramedullary alignment of the tibial component in the Triathlon knee. Journal of orthopaedic surgery and research. 2011;6(1):44.

11. Brys DA, Lombardi JA, Mallory TH, Vaughn BK. A comparison of intramedullary and extramedullary alignment systems for tibial component placement in total knee arthroplasty. Clinical orthopaedics and related research. 1991(263):175-9. 
12. Rezende BdRM, Fuchs T, Nishi RN, Hatem MA, da Silva LMF, Fuchs R, et al. Alignment of the tibial component in total knee arthroplasty procedures using an intramedullary or extramedullary guide: double-blind randomized prospective study. Revista Brasileira de Ortopedia (English Edition). 2015;50(2):168-73.

13. La Floresta P, Napolitano C, Covatta V, Gennarelli A, editors. A COMPARISON OF INTRAMEDULLARY AND EXTRAMEDULLARY ALIGNMENT SYSTEMS FOR TIBIAL COMPONENT PLACEMENT IN TOTAL KNEE ARTHROPLASTY. Orthopaedic Proceedings; 2006: The British Editorial Society of Bone \& Joint Surgery.

14. Tang Q, Shang P, Zheng G, Xu H-Z, Liu H-X. Extramedullary versus intramedullary femoral alignment technique in total knee arthroplasty: a meta-analysis of randomized controlled trials. Journal of orthopaedic surgery and research. 2017;12(1):82.

15. Blakeney WG, Khan RJ, Palmer JL. Functional outcomes following total knee arthroplasty: a randomised trial comparing computer-assisted surgery with conventional techniques. The Knee. 2014;21(2):364-8.

16. Chin PL, Yang KY, Yeo SJ, Lo NN. Randomized control trial comparing radiographic total knee arthroplasty implant placement using computer navigation versus conventional technique. The Journal of arthroplasty. 2005;20(5):618-26.

17. Rottman SJ, Dvorkin M, Gold D. Extramedullary versus intramedullary tibial alignment guides for total knee arthroplasty. Orthopedics. 2005;28(12):1445-8.

18. de Kroon KE, Houterman S, Janssen RP. Leg alignment and tibial slope after minimal invasive total knee arthroplasty: a prospective, randomized radiological study of intramedullary versus extramedullary tibial instrumentation. The Knee. 2012;19(4):270-4.

19. Blakeney WG, Khan RJ, Wall SJ. Computer-assisted techniques versus conventional guides for component alignment in total knee arthroplasty: a randomized controlled trial. JBJS. 2011;93(15):1377-84.

20. Compton J, Owens J, Otero J, Noiseux N, Brown T. Extramedullary Guide Alignment Is not Affected by Obesity in Primary Total Knee Arthroplasty. The journal of knee surgery. 2020.

\section{Figures}




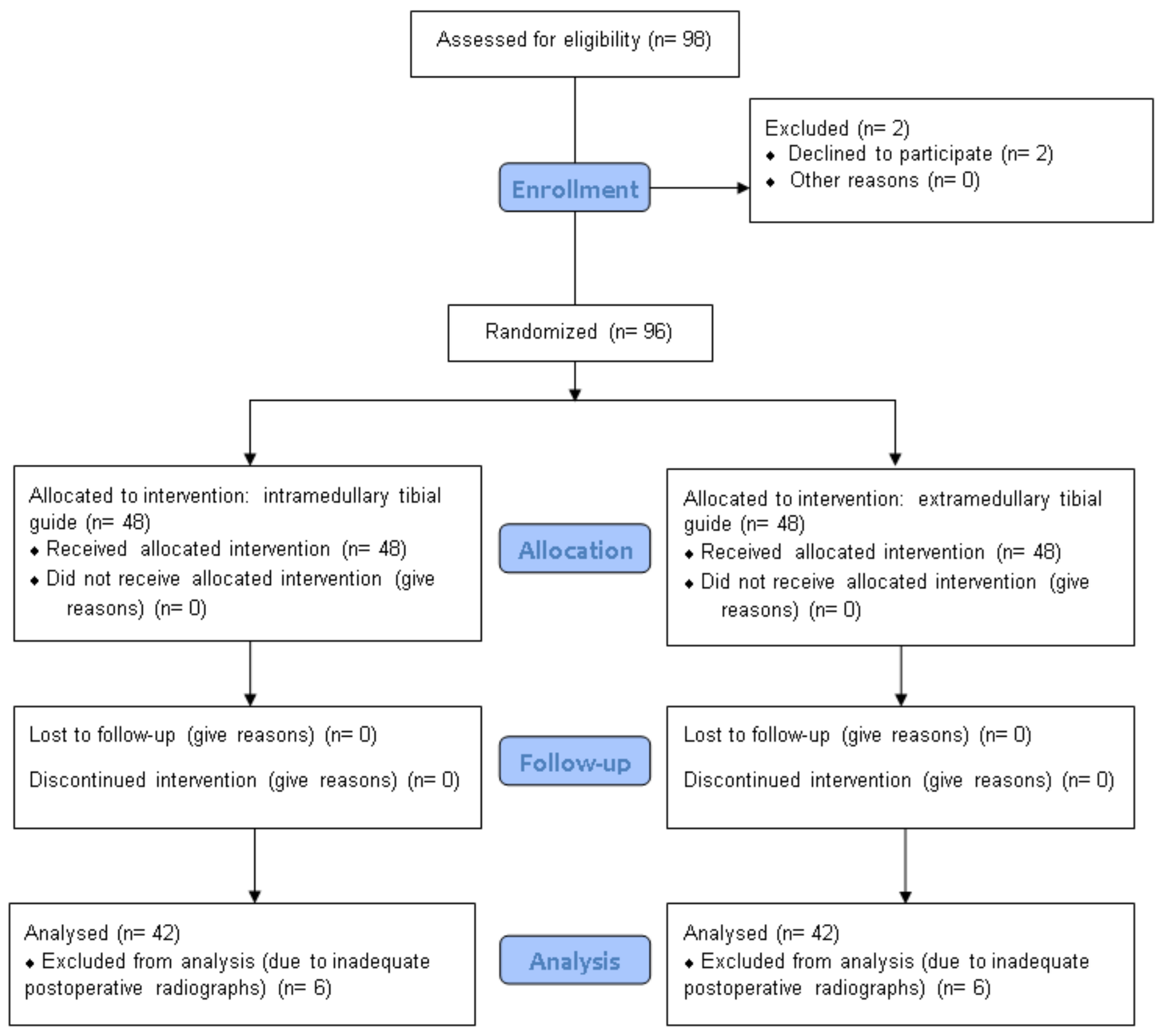

\section{Figure 1}

The CONSORT flowchart of the study 


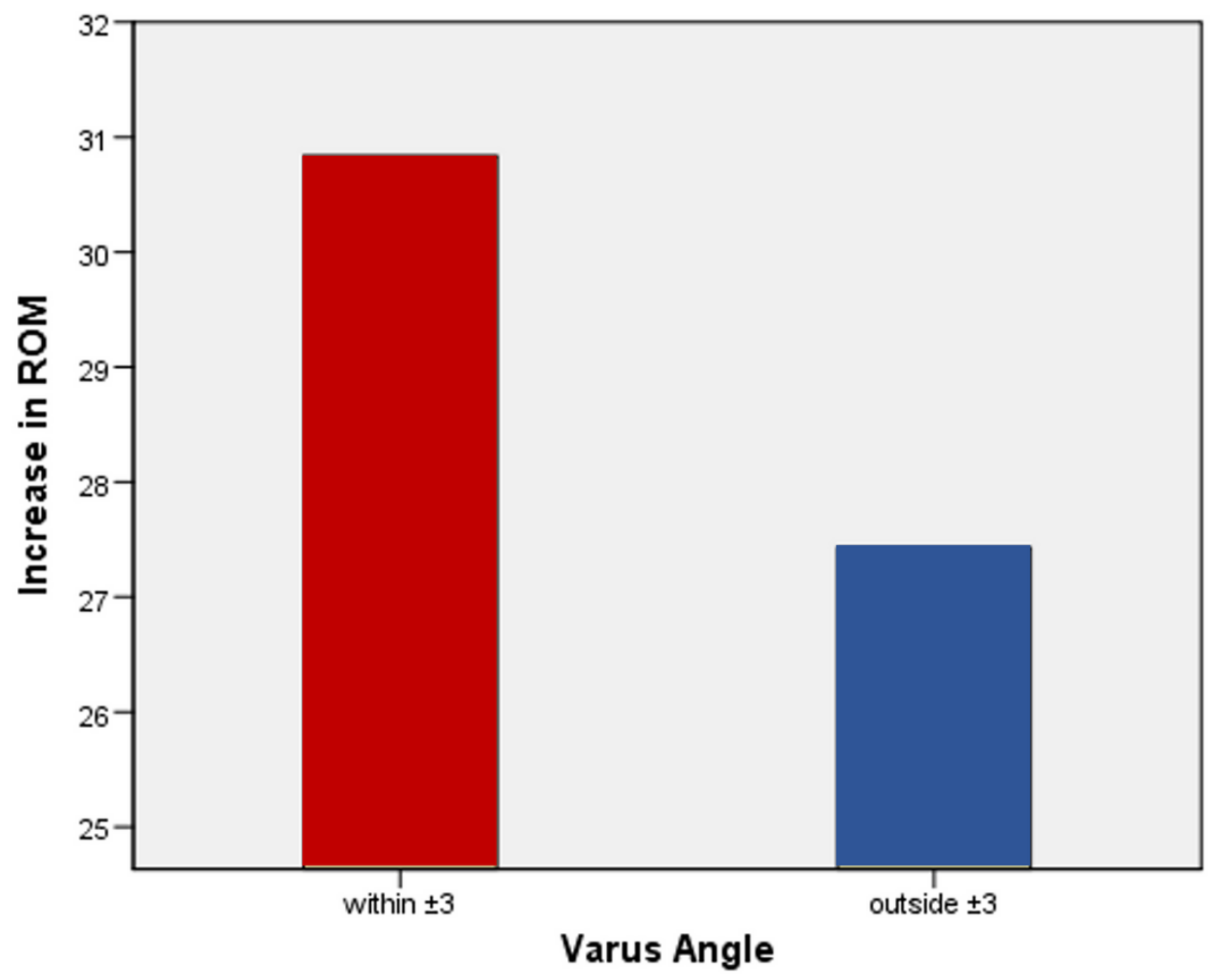

Figure 2

The increase in knee ROM in patients with a postoperative VA within $\pm 3^{\circ}$ of normal vs. those outside this range at the mean follow-up of 13 months 